\title{
Solving model of mooring system
}

\author{
Chenyang Li \\ School of North China Electric Power University, Baoding 071000, China \\ 1572371776@qq.com
}

Keywords: Mooring system, Iterative method, optimal design parameters

\begin{abstract}
In this paper, the design of mooring system in transmission node of near shallow water observation network is studied, and through the static analysis of the equilibrium state of the mooring system, we establish the relationship between the components based on iterative method. Then we can establish the goal programming model according to the system performance and reliability requirements in order to get the optimal design parameters.
\end{abstract}

\section{Introduction}

The transmission nodes of near shallow water observation network are composed of buoy system, mooring system and underwater acoustic communication system, and mooring system is composed of a steel pipe, steel drums, heavy ball, welding anchor and special anti drag anchor. When the steel drums is vertical, acoustic communication equipment work best. If the drum tilts, the equipment working effect will be influenced. And the tilt angle (the angle between the steel drums and vertical lines) more than 5 degrees, equipment perform worst. Also because of the importance of buoys, it's necessary to analyze the distribution of the buoy system and optimize the design parameter of it.

\section{Model preparation}

\subsection{Hypothesis.}

1. The components of a mooring system are connected in an articulated manner, and the joints do not limit the freedom of movement of the components

2. Steel drums uniform mass distribution, gravity and centroid coincidence

3. The connecting points of each component are located at the center of the adjacent element surface

2.2 Variable assumptions.

\begin{tabular}{cc}
\hline \hline symbol & instruction \\
\hline \hline$\theta_{i}$ & The angle between the central axis of length direction of the steel pipe and sea level \\
$b_{0}$ & The length of each link in the chain \\
$m_{0}$ & The weight of link of chain \\
$\beta_{i}$ & The angle between the central axis of length direction of the link and sea level \\
$F_{x}$ & The force of the connector in the direction of the $x$ axis \\
$F_{y}$ & The force of the connector in the direction of the $y$ axis \\
$F_{\text {float }}$ & The buoyancy of the connecting piece \\
\hline \hline
\end{tabular}

\subsection{Problem analysis.}

The analysis of the mooring system shows that, the final equilibrium state of mooring system composed of these objects in the wind under the influence of outside is certain. Then we can choose the final equilibrium state of the mooring system as the object of study under the condition of wind speed stability. Under the premise of assumption, each component can be used as the research object to analyze their respective forces. And the force of each object can be reduced to a plane perpendicular 
to sea level, we can break down the force at the joint into two components along the axis of the coordinate system $^{[1]}$. Finally, according to the equilibrium condition, the force relation of the object can be expressed.

\section{Model building}

\subsection{Analysis of each connector.}

In order to determine the state of each part of the mooring system, first of all, the force of the connecting parts should be determine, so we need establish the equation of force equilibrium state of connections. Here we take the seabed plane as the $x$ axis and parallel with the anchor chain as the $y$ axis.

Mechanical equilibrium equation: $\left\{\begin{array}{l}\sum F_{x}=0 \\ \sum F_{y}=0\end{array}, \quad \sum M_{o}=0\right.$

(1) Force analysis of buoy

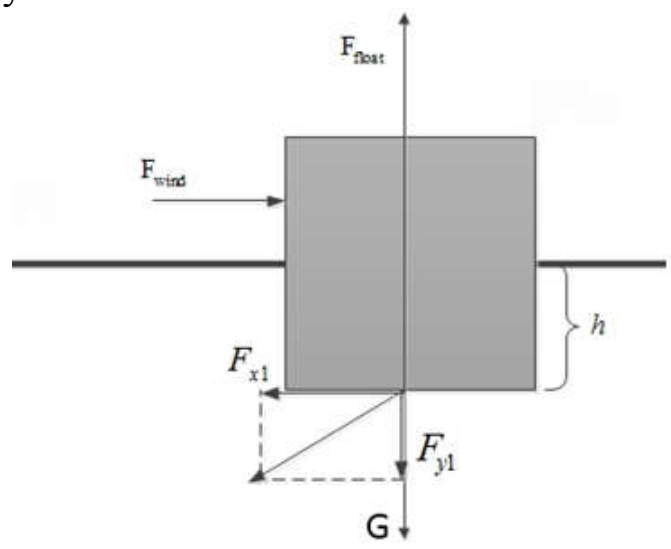

Fig. 1 Force diagram of buoy

The mechanical balance equation of buoy can be built from the figure above:

$\left\{\begin{array}{l}F_{\text {wind }}-F_{x 1}=0 \\ G-F_{\text {float }}+F_{y 1}=0\end{array}\right.$

(2) Force analysis of steel pipe:

The connection between the different steel pipe is hinged, and the relationship is force and the reaction force between the steel pipe and the two sides of the connecting objects. For the first section of the steel pipe, The force is shown below:



Fig. 2 Force diagram of the first one steel pipe

So we can get the balance equation: 


$$
\left\{\begin{array}{l}
F_{x 1}-F_{x 2}=0 \\
F_{y 1}-F_{y 2}+F_{\text {float }}+G=0 \\
0.5 l_{0}\left(F_{y 1}+F_{y 2}\right) \cos \theta_{1}-0.5 l_{0}\left(F_{x 1}+F_{x 2}\right) \sin \theta_{1}=0
\end{array}\right.
$$

The same is true for the second steel tubes in the middle.

(3) Force analysis of steel drums:
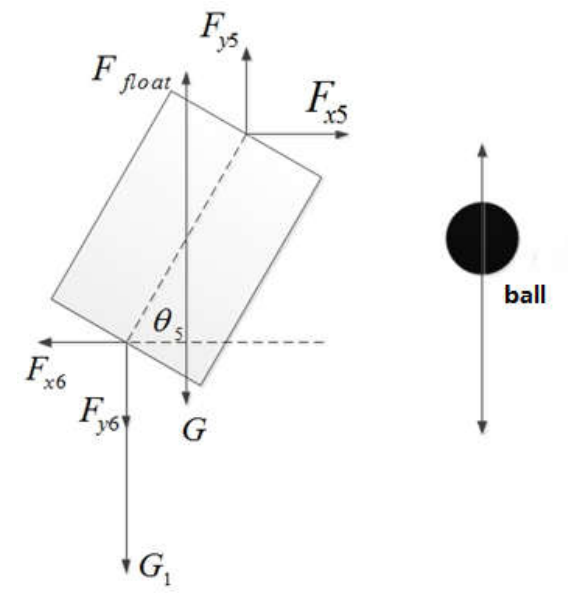

Fig. 3 Steel drums

$$
\left\{\begin{array}{l}
F_{y 5}+F_{\text {float }}-G-G_{1}-F_{y 6}=0 \\
F_{x 5}-F_{x 6}=0 \\
0.5 l_{1}\left(F_{y 5}+F_{y 6}\right) \cos \theta_{5}-0.5 l_{1}\left(F_{x 5}+F_{x 6}\right) \sin \theta_{5}+0.5 G_{1} \cos \theta_{5}=0
\end{array}\right.
$$

(4) Force analysis of anchor chain:

In actual use, the overall length of the chain is much larger than the length of a single link. In considering the overall force of the chain link, the mass distribution of the link is even, and the center of gravity is concentrated at $1 / 2$ of the chain length. The anchor chain can be regarded as a quasi static model similar to the catenary model ${ }^{[2]}$.

$$
\left\{\begin{array}{l}
F_{y 6}+F_{\text {float }}-F_{y 7}-G=0 \\
F_{x 6}-F_{x 7}=0
\end{array}\right.
$$

We define the inclination of the end chain of the anchor chain as the tangent direction at the link between the anchor end and the anchor. Through node analysis method above, we have established mechanical equilibrium equation of connecting piece. And given the wind speed, the length of the anchor chain, the depth of the sea water and the weight of the heavy ball, the position of the connections in the mooring system can be uniquely determined. In order to meet the restriction of mooring system at given sea water depth, the new constraint conditions are established with the vertical height of each connecting piece equal to the depth of sea water. The relationship is as follows:

$$
\sum_{i=1}^{n} b_{0} \sin \beta_{i}+l_{1} \sin \theta_{5}+\sum_{j=1}^{4} l_{0} \sin \theta_{j}+h=H
$$

In which, $n$ represents the number of links in the chain.

Finally, we need to determine the state of each link and take a single link as the object of study. 


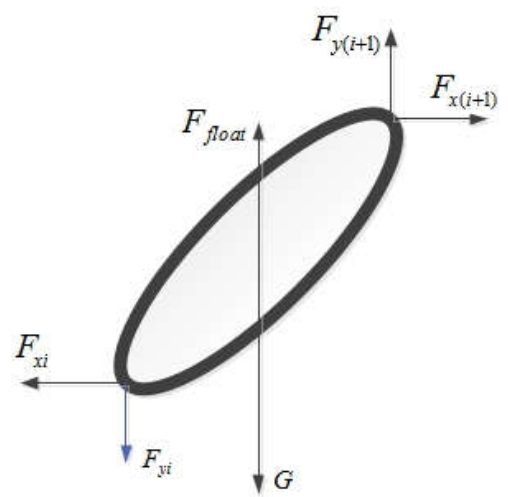

Fig. 4 Single link of anchor chain

$\left\{\begin{array}{l}F_{x(i+1)}-F_{x i}=0 \\ F_{y(i+1)}+F_{\text {float }}-G-F_{y i}=0 \\ 0.5 m_{0} g\left(F_{y(i+1)}+F_{y i}\right) \cos \beta_{i}-0.5 m_{0} g\left(F_{x(i+1)}+F_{x i}\right) \sin \beta_{i}=0\end{array}\right.$

From the bottom end of the chain link with the anchor, and according to recurrence relation, we can solve the mooring system balance.

\subsection{Example of using our model.}

Here we select an actual system to solve a problem and verify the effectiveness of this model. The length of anchor chain is $22.05 \mathrm{~m}$, the number of link is 210 , wind speed is $2 \mathrm{~m} / \mathrm{s}$, the water depth is $18 \mathrm{~m}$, the weight of ball is $1200 \mathrm{~kg}$.

We mainly analyze the influence of wind speed on the main parameters of mooring system, we select the wind speed for $14 \mathrm{~m} / \mathrm{s}, 16 \mathrm{~m} / \mathrm{s}, 18 \mathrm{~m} / \mathrm{s}, 20 \mathrm{~m} / \mathrm{s}, 22 \mathrm{~m} / \mathrm{s}, 24 \mathrm{~m} / \mathrm{s}$, in addition to solve the problem, the curve of the shape of the chain as shown below:

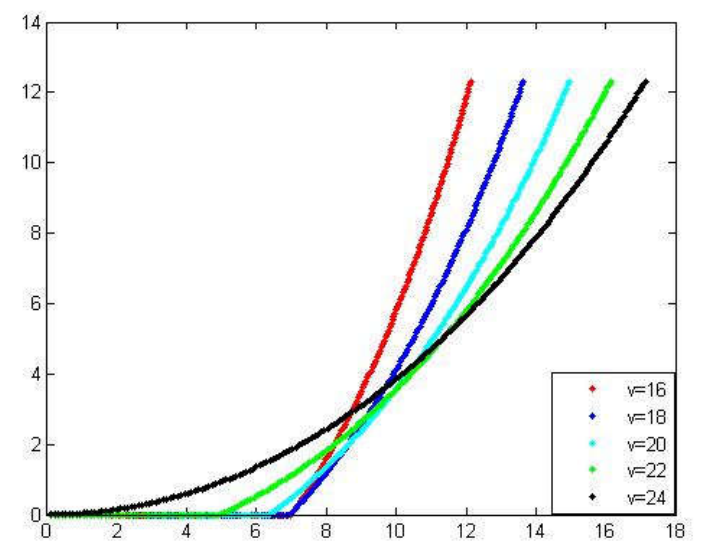

Fig. 5 The shape of anchor chain

This shows that the model can reflect the actual distribution of the anchor chain. It is also highly consistent with practice, and the model is more practical. Therefore, when we solve the equilibrium state of the system, it can ensure that the system is in good condition, also help us to determine the parameters of each part of the system.

\section{References}

[1]. Yanbai Zhou. A course in theoretical mechanics [M].Beijing: Higher Education Press, 1986

[2]. Yougang Tang, Shuxia Zhang, Ruoyu Zhang, Haixiao Liu. Advances in research on dynamic characteristics of deep sea mooring system [J]. Oceanographic engineering, 2008, 01:120-126;

[3]. Yazhou Wang. Study on mooring system of deep sea single point mooring ocean buoy [D]. Ocean University of China, 2013 
[4]. Fei Guo, Yanfeng Sheng, Honghui He, Feng Jiang, Jingxiang Zhang. Research on buoy and buoy mooring system for shallow water environment observation[J]. Marine technology, 2000, 02: $7-12$ 